\title{
Phase diagrams of the Bose-Hubbard model at finite temperature
}

\author{
I.V. Stasyuk, T.S. Mysakovych \\ Institute for Condensed Matter Physics of the National Academy of Sciences of Ukraine, \\ 1 Svientsitskii Str., 79011 Lviv, Ukraine
}

Received July 8, 2009

\begin{abstract}
The phase transitions in the Bose-Hubbard model are investigated. A single-particle Green's function is calculated in the random phase approximation and the formalism of the Hubbard operators is used. The regions of existence of the superfluid and Mott insulator phases are established and the $(\mu, t)$ (the chemical potential transfer parameter) phase diagrams are built. The effect of temperature change on this transition is analyzed and the phase diagram in the $(T, \mu)$ plane is constructed. The role of thermal activation of the ion hopping is investigated by taking into account the temperature dependence of the transfer parameter. The reconstruction of the Mott-insulator lobes due to this effect is analyzed.
\end{abstract}

Key words: Bose-Hubbard model, phase transition

PACS: 03.75.Lm, 05.30.Jp

\section{Introduction}

The Bose-Hubbard model (BHM) has been intensively investigated in the last 15 years. This model is of great interest due to the experimental realization of Bose-Einstein condensation of ultra-cold atoms in optical lattices (see, for example, [1]). Various theoretical methods were used to study the model: mean-field theory [2], random phase approximation [3,4], strong coupling approach [5], quantum Monte-Carlo method [6], bosonic version of dynamical mean field theory [7]. The Hamiltonian of the model includes two terms. One is connected with the on-site energy $U$, which describes repulsion of bosons at a lattice site; another one describes the nearest-neighbor hopping with tunneling parameter $t$ :

$$
H=-\sum_{i j} t_{i j} b_{i}^{+} b_{j}+\frac{U}{2} \sum_{i} n_{i}\left(n_{i}-1\right)-\mu \sum_{i} n_{i},
$$

$\mu$ is the chemical potential. The competition between the kinetic and on-site repulsion terms defines the equilibrium state of the system. The existence of the superfluid and Mott-insulator phases is a characteristic feature of this model. When the kinetic energy dominates $(t / U \gg 1)$ the ground state of the system is superfluid (SF). In the opposite case the ground state is a Mott insulator (MI).

In the limit $U \rightarrow \infty$ this model reduces to the hard-core boson model. Models of a lattice gas type with Pauli statistics of particles are often used for the description of ionic conductors and calculation of their conductivity starting from the works of Mahan [8] and others [9,10]. Recently the one-particle spectrum has been investigated in the one-dimensional limit [10]. As noted above, the Bose-Hubbard model can be directly applied to the description of optical lattices. Besides, the model of Bose-Hubbard type can be also useful not only for investigation of ionic conductivity in crystalline ionic conductors, but it can also be applied to description of the intercalation in crystals [11] and kinetics of ionic adsorption on the crystal surfaces [12].

In this work we consider the case of the finite value of the on-site interaction $U$ and study phase transitions in the BHM at finite temperatures. Most of the previous investigations by the BoseHubbard model were restricted to the case of zero temperature. Recently only the low-temperature 
case $(T \ll U)$ has been investigated [3,13]. A special attention will be paid here to the investigation of the effect of thermal activation of the ion hopping on the shape of phase diagrams (we take into account the temperature dependence of the transfer parameter). The effect of thermal activation can be important in the case of ionic conductors. It was not studied in the framework of the BHM; such an investigation has been performed only in the hard-core boson limit [14] so far.

\section{The random phase approximation}

We introduce the on-site basis $\mid n\left\langle_{i}\right.$ ( $n$ is a number of bosons on a site $i$ ) and use the Hubbard operators $X_{i}^{n m}=|n\rangle_{i}\left\langle\left. m\right|_{i}\right.$. The Bose-operators of creation and annihilation can be expressed in terms of the $\mathrm{X}$-operators:

$$
b_{i}=\sum_{n} \sqrt{n+1} X_{i}^{n, n+1}, \quad n_{i}=\sum_{n} n X_{i}^{n n} .
$$

Then the Hamiltonian (1) can be written as follows:

$$
\begin{aligned}
H & =H_{0}+H_{1}, \\
H_{0} & =\sum_{i n} \lambda_{n} X_{i}^{n n} ; \quad \lambda_{n}=\frac{U}{2} n(n-1)-\mu n, \\
H_{1} & =-\sum_{i j} t_{i j} b_{i}^{+} b_{j} .
\end{aligned}
$$

To find the Green's function $\left\langle\left\langle b \mid b^{+}\right\rangle\right\rangle$we employ the equation of motion technique:

$$
\begin{aligned}
\omega\left\langle\left\langle X_{l}^{m, m+1} \mid X_{p}^{r+1, r}\right\rangle\right\rangle & =\frac{1}{2 \pi} \delta_{l p} \delta_{m r}\left\langle X_{l}^{m r}-X_{l}^{r+1, m+1}\right\rangle+\left(\lambda_{m+1}-\lambda_{m}\right)\left\langle\left\langle X_{l}^{m, m+1} \mid X_{l}^{r+1, r}\right\rangle\right\rangle \\
& -\sum_{i j} t_{i j} \sqrt{m+1} \delta_{l i}\left\langle\left\langle\left(X_{l}^{m m}-X_{l}^{m+1, m+1}\right) b_{j} \mid X_{p}^{r+1, r}\right\rangle\right\rangle \\
& -\sum_{i j} t_{i j} \delta_{l j}\left\langle\left\langle b_{i}^{+}\left(\sqrt{m+2} X_{l}^{m, m+2}-\sqrt{m} X_{l}^{m-1, m+1}\right) \mid X_{p}^{r+1, r}\right\rangle\right\rangle
\end{aligned}
$$

We use the random phase approximation (which is an analogy to the Hubbard-I approximation for the case of the fermionic Hubbard model) and perform the following decoupling:

$$
\begin{aligned}
\left\langle\left\langle\left(X_{l}^{m, m}-X_{l}^{m+1, m+1}\right) b_{j} \mid X_{p}^{r+1, r}\right\rangle\right\rangle & \approx\left\langle X^{m m}-X^{m+1, m+1}\right\rangle\left\langle\left\langle b_{j} \mid X_{p}^{r+1, r}\right\rangle\right\rangle, \\
\left\langle\left\langle b_{i}^{+} X_{l}^{m, m+2} \mid X_{p}^{r+1, r}\right\rangle\right\rangle & \approx 0 .
\end{aligned}
$$

It should be noted that here we consider the Mott-insulator phase, where $\left\langle b_{i}\right\rangle=0$. If we want to investigate the superfluid phase we should take into account that in this phase $\left\langle b_{i}\right\rangle \neq 0$ (this case is considered below).

As a result, the following expression for the Green's function is obtained:

$$
\begin{aligned}
& \left\langle\left\langle X_{l}^{m, m+1} \mid X_{p}^{r+1, r}\right\rangle\right\rangle=\frac{1}{2 \pi} \delta_{l p} \delta_{m r} \frac{Q_{m}}{\omega-\Delta_{m}}-\frac{Q_{m}}{\omega-\Delta_{m}} \sqrt{m+1} \sum_{j} t_{l j}\left\langle\left\langle b_{j} \mid X_{p}^{r+1, r}\right\rangle\right\rangle, \\
& \Delta_{m}=\lambda_{m+1}-\lambda_{m}, \quad\left\langle X^{m m}-X^{m+1, m+1}\right\rangle=Q_{m} .
\end{aligned}
$$

We pass to $\boldsymbol{k}$-representation and the Green's function $G_{\boldsymbol{k}}(\omega) \equiv\left\langle\left\langle b \mid b^{+}\right\rangle\right\rangle_{\boldsymbol{k} \omega}$ can be written as

$$
\begin{aligned}
G_{\boldsymbol{k}}(\omega) & =\frac{1}{2 \pi} \frac{g^{0}}{1+g^{0} t_{\boldsymbol{k}}}, \\
g^{0}(\omega) & =\sum_{m} \frac{Q_{m}(m+1)}{\omega-\Delta_{m}} .
\end{aligned}
$$


We should note that in the independent subband approximation (this approximation is valid in the case $U \gg t$ and in the regions of the integer value of $\mu / U)$ the Green's function takes the form

$$
G_{\boldsymbol{k}}=\frac{1}{2 \pi} \sum_{m} \frac{(m+1) Q_{m}}{\omega-\Delta_{m}+t_{\boldsymbol{k}}(m+1) Q_{m}},
$$

and the subband energy is equal to $\lambda_{m}=\Delta_{m}-(m+1) Q_{m} t_{\boldsymbol{k}}$.

In order to study the phase with the Bose-Einstein condensate we can also apply the decoupling of the (5) type, which in this case is more complicated:

$$
\begin{aligned}
\left\langle\left\langle\left(X_{l}^{m, m}-X_{l}^{m+1, m+1}\right) b_{j} \mid X_{p}^{r+1, r}\right\rangle\right\rangle \approx & Q_{m}\left\langle\left\langle b_{j} \mid X_{p}^{r+1, r}\right\rangle\right\rangle \\
& +\left\langle b_{j}\right\rangle\left\langle\left\langle\left(X_{l}^{m m}-X_{l}^{m+1, m+1}\right) \mid X_{p}^{r+1, r}\right\rangle\right\rangle .
\end{aligned}
$$

The function $\left\langle\left\langle\left(X_{l}^{m, m}-X_{l}^{m+1, m+1}\right) b_{j} \mid X_{p}^{r+1, r}\right\rangle\right\rangle$ satisfies the following equation:

$$
\begin{gathered}
\omega\left\langle\left\langle X_{l}^{m, m}-X_{l}^{m+1, m+1} \mid X_{p}^{r+1, r}\right\rangle\right\rangle=\frac{1}{2 \pi} \delta_{l p}\left(\left\langle X^{m, m-1}\right\rangle \delta_{m, r+1}+\left\langle X^{m+2, m+1}\right\rangle \delta_{m+1, r}\right. \\
\left.-2\left\langle X^{m+1, m}\right\rangle \delta_{m r}\right)-\sum_{j} t_{l j}\left(\sqrt{m}\left\langle\left\langle X_{l}^{m, m-1} b_{j}-b_{j}^{+} X_{l}^{m-1, m} \mid X_{p}^{r+1, r}\right\rangle\right\rangle\right. \\
+\sqrt{m+2}\left\langle\left\langle X_{l}^{m+2, m+1} b_{j}-b_{j}^{+} X_{l}^{m+1, m+2} \mid X_{p}^{r+1, r}\right\rangle\right\rangle \\
\left.-2 \sqrt{m+1}\left\langle\left\langle X_{l}^{m+1, m} b_{j}-b_{j}^{+} X_{l}^{m, m+1} \mid X_{p}^{r+1, r}\right\rangle\right\rangle\right) .
\end{gathered}
$$

Using decoupling (10) in the independent subband approximation (which is equivalent to taking into consideration the transition between $|m\rangle$ and $|m+1\rangle$ levels only) the equation takes a simpler form

$$
\begin{aligned}
& \omega\left\langle\left\langle X_{l}^{m, m}-X_{l}^{m+1, m+1} \mid X_{p}^{r+1, r}\right\rangle\right\rangle=-\frac{1}{\pi} \delta_{l p} \delta_{m r}\left(\left\langle X^{m+1, m}\right\rangle\right. \\
& +2(m+1) \sum_{j} t_{l j}\left(\left\langle X_{j}^{m, m+1}\right\rangle\left\langle\left\langle X_{l}^{m+1, m} \mid X_{p}^{r+1, r}\right\rangle\right\rangle-\left\langle X_{l}^{m, m+1}\right\rangle\left\langle\left\langle X_{j}^{m+1, m} \mid X_{p}^{r+1, r}\right\rangle\right\rangle\right) \\
& \quad-2(m+1) \sum_{j} t_{l j}\left(\left\langle X_{j}^{m+1, m}\right\rangle\left\langle\left\langle X_{l}^{m, m+1} \mid X_{p}^{r+1, r}\right\rangle\right\rangle-\left\langle X_{l}^{m+1, m}\right\rangle\left\langle\left\langle X_{j}^{m, m+1} \mid X_{p}^{r+1, r}\right\rangle\right\rangle\right) .
\end{aligned}
$$

In a similar way, we can obtain equations for the Green's functions $\left\langle\left\langle X_{l}^{m+1, m} \mid X_{p}^{r+1, r}\right\rangle\right\rangle$ and $\left\langle\left\langle X_{l}^{m, m+1} \mid X_{p}^{r+1, r}\right\rangle\right\rangle:$

$$
\begin{gathered}
\left(\omega+\Delta_{m}\right)\left\langle\left\langle X_{l}^{m+1, m} \mid X_{p}^{r+1, r}\right\rangle\right\rangle=(m+1) \sum_{j} t_{l j} Q_{m}\left\langle\left\langle X_{j}^{m+1, m} \mid X_{p}^{r+1, r}\right\rangle\right\rangle \\
+(m+1) \sum_{j} t_{l j}\left\langle X_{j}^{m+1, m}\right\rangle\left\langle\left\langle X_{l}^{m, m}-X_{l}^{m+1, m+1} \mid X_{p}^{r+1, r}\right\rangle\right\rangle, \\
\left(\omega-\Delta_{m}\right)\left\langle\left\langle X_{l}^{m, m+1} \mid X_{p}^{r+1, r}\right\rangle\right\rangle=\frac{1}{2 \pi} \delta_{l p} \delta_{m r} Q_{m}-(m+1) \sum_{j} t_{l j} Q_{m}\left\langle\left\langle X_{j}^{m, m+1} \mid X_{p}^{r+1, r}\right\rangle\right\rangle \\
-(m+1) \sum_{j} t_{l j}\left\langle X_{j}^{m, m+1}\right\rangle\left\langle\left\langle\left(X_{l}^{m, m}-X_{l}^{m+1, m+1}\right) \mid X_{p}^{r+1, r}\right\rangle\right\rangle .
\end{gathered}
$$

The levels $|m\rangle,|m+1\rangle$ form a two-state subspace where we can introduce pseudospin formalism:

$$
X_{l}^{m, m+1}=S_{l, m}^{-}, \quad X_{l}^{m+1, m}=S_{l, m}^{+}, \quad \frac{1}{2}\left\langle X_{l}^{m+1, m+1}-X_{l}^{m m}\right\rangle=S_{l, m}^{z} .
$$


The mean value $Q_{m}=-2\left\langle S_{m}^{z}\right\rangle$ is connected with the longitudinal component of the pseudospin, while the order parameter $\left\langle b_{j}\right\rangle$ in the condensate phase is proportional to the transverse component $\left\langle b_{j}\right\rangle \approx \sqrt{m+1}\left\langle S_{m}^{-}\right\rangle=\sqrt{m+1}\left\langle S_{m}^{x}\right\rangle$.

The set of equations (12), (13), and (14) can be rewritten using pseudospin Green's functions. The equations are analogous to those obtained in the RPA for the XXZ model. Their solution can be written in the form given in [14]

$$
\left\langle\left\langle S_{m}^{+} \mid S_{m}^{-}\right\rangle\right\rangle=\frac{1}{2 \pi}\left\langle\sigma_{m}^{z}\right\rangle \frac{E_{m}\left(\cos ^{2} \theta_{m}+1\right)+2 \omega \cos \theta_{m}-4\left\langle\sigma_{m}^{z}\right\rangle J_{q, m} \cos ^{2} \theta_{m}}{\omega^{2}-\left(E_{m}-2\left\langle\sigma_{m}^{z}\right\rangle J_{q, m}\right)\left(E_{m}-2\left\langle\sigma_{m}^{z}\right\rangle J_{q, m}\right) \cos ^{2} \theta_{m}} .
$$

In the mean field approximation the angle $\theta_{m}$ and parameter $\left\langle\sigma_{m}^{z}\right\rangle$ are defined by the following equations:

$$
\begin{aligned}
& \Delta_{m} \sin \theta_{m}-2(m+1) t_{0}\left\langle S_{m}^{x}\right\rangle \cos \theta_{m}=0 \\
& \left\langle\sigma_{m}^{z}\right\rangle=\frac{1}{2} \tanh \frac{\beta E_{m}}{2}, \quad E_{m}=\sqrt{\Delta_{m}^{2}+4(m+1)^{2} t_{0}^{2}\left\langle S_{m}^{x}\right\rangle^{2}} \\
& t_{0} \equiv t_{q=0}, \quad J_{q, m}=(m+1) t_{q}, \quad\left\langle S_{m}^{x}\right\rangle=-\left\langle\sigma_{m}^{z}\right\rangle \sin \theta_{m}
\end{aligned}
$$

The solution $\sin \theta_{m}=0\left(\left\langle S_{m}^{x}\right\rangle=0\right)$ corresponds to the normal (nonsuperfluid) phase; in the phase with condensate $\cos \theta_{m}=-\frac{\Delta_{m}}{2(m+1) t_{0}\left\langle\sigma_{m}^{z}\right\rangle}$ and $\left\langle\sigma_{m}^{z}\right\rangle$ is given by the solution of the following equation

$$
\left\langle\sigma_{m}^{z}\right\rangle=\frac{1}{2} \tanh \left(\beta(m+1) t_{0}\left\langle\sigma_{m}^{z}\right\rangle\right)
$$

The transition to the phase with condensate takes place at temperature

$$
T_{m}^{\mathrm{c}}=\Delta_{m}\left(\ln \frac{1+\frac{\Delta_{m}}{J_{0, m}}}{1-\frac{\Delta_{m}}{J_{0, m}}}\right)^{-1}
$$

This temperature reaches its maximum value $T_{\max }^{\mathrm{c}}=J_{0, m} / 2$ at $\Delta_{m}=m U-\mu=0$.

The case of pair of the states $|0\rangle,|1\rangle$ corresponds to the hard core boson model; the peculiarities of the spectrum and density of states were investigated in [14]. The thermodynamics of the model was also studied in [14] in the case of the thermal activated hopping.

\section{Results}

The phase transition from the Mott-insulator to superfluid phase is characterized by divergence of the Green's function $G_{\boldsymbol{k}=0}(\omega=0) \rightarrow \infty$. This condition can be obtained using the following reasoning. Let us find the expression for the commutator $\left[X_{l}^{m, m+1}, H\right]$ in the Hubbard-I type approximation:

$$
\left[X_{l}^{m, m+1}, H\right] \approx \Delta_{m} X_{l}^{m, m+1}-\sum_{j} t_{l j} \sqrt{m+1}\left\langle X^{m m}-X^{m+1, m+1}\right\rangle b_{j} .
$$

The mean value of this commutator should be equal to zero (because the average $\left\langle X^{m, m+1}\right\rangle$ does not depend on time in a stationary state). After decoulping we get:

$$
\left\langle b_{\boldsymbol{k}}\right\rangle\left[1-t_{\boldsymbol{k}} \sum_{m} \frac{m+1}{\Delta_{m}} Q_{m}\right]=0
$$

Thus, we can see that the nonzero solution $\left\langle b_{\boldsymbol{k}}\right\rangle \neq 0$ appears in the point of divergence of the Green's function $G_{\boldsymbol{k}}(\omega=0) \rightarrow \infty$ (8). The condition (22) in the case $t>0$ (considered in this paper) is fulfilled at the center of the Brillouin zone when $k=0$ (an uniform phase case) and $G_{\boldsymbol{k}=0}(\omega=0) \rightarrow \infty$ in the phase transition point. In the case $t<0$ this condition is satisfied at the Brillouin zone edge when $\boldsymbol{k}=(\pi, \pi, \ldots)$ (a modulation phase case). 
In our numerical calculations we used the diagonal Hamiltonian $H_{0}$ to calculate the averages $\left\langle X^{m m}\right\rangle$. In this case the condition (22) coincides with that obtained in [13] in the framework of the diagrammatic hopping expansion at the calculation of temperature Green's functions (the first order in the self-energy in the notations used in [13]).

Let us consider the effect of thermal activation. We rewrite the parameter of the ion hopping in the following form: $t=t_{0} \exp (-\beta \Delta)$. Such an approximation was used in [8,14] to take into account the presence (in ionic conductors) of a static barrier over which a particle should hop. It can also be obtained as a result of renormalization due to the interaction with phonons.
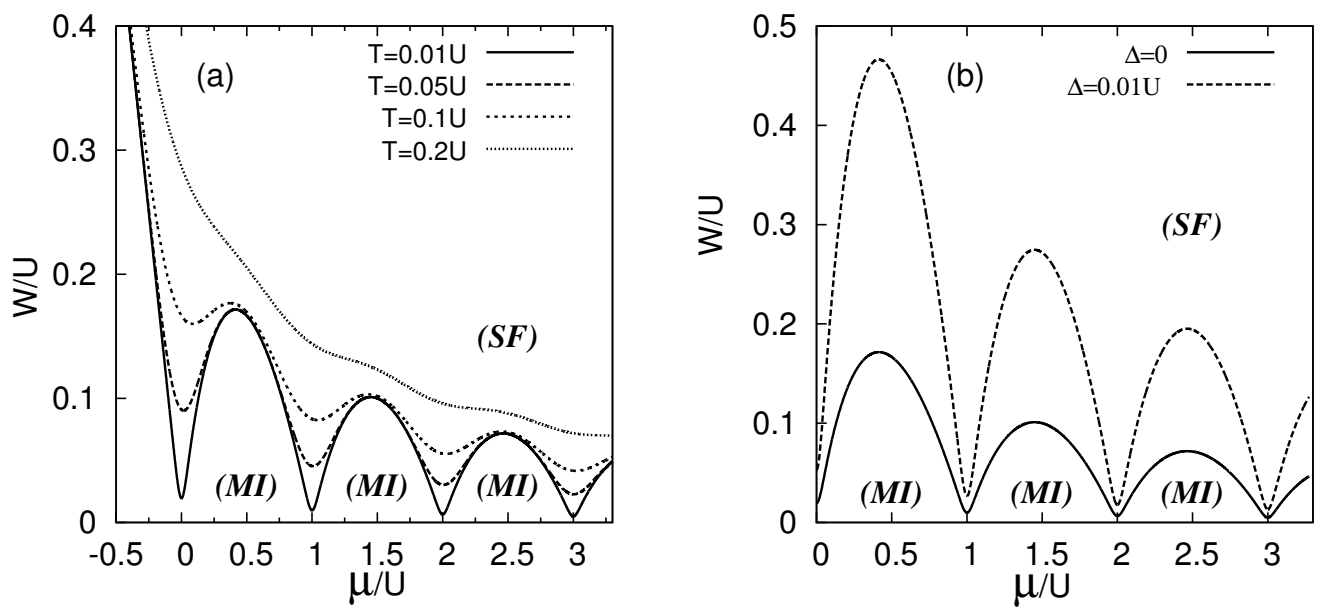

Figure 1. The $(W, \mu)$ phase diagram at $\Delta=0$ (a) and $\Delta \neq 0(\mathrm{~b})$.

In figure 1 the $(W, \mu)$ phase diagram is shown ( $W$ is a half width of the initial energy band, $-W<t<W)$. The system can pass from the MI to SF phase at the change of the chemical potential as well as at the change of the transfer parameter. The effect of thermal activation on phase transitions is illustrated in figure 1b. Thermal activation leads to the expansion of the region of the Mott-insulator phase.
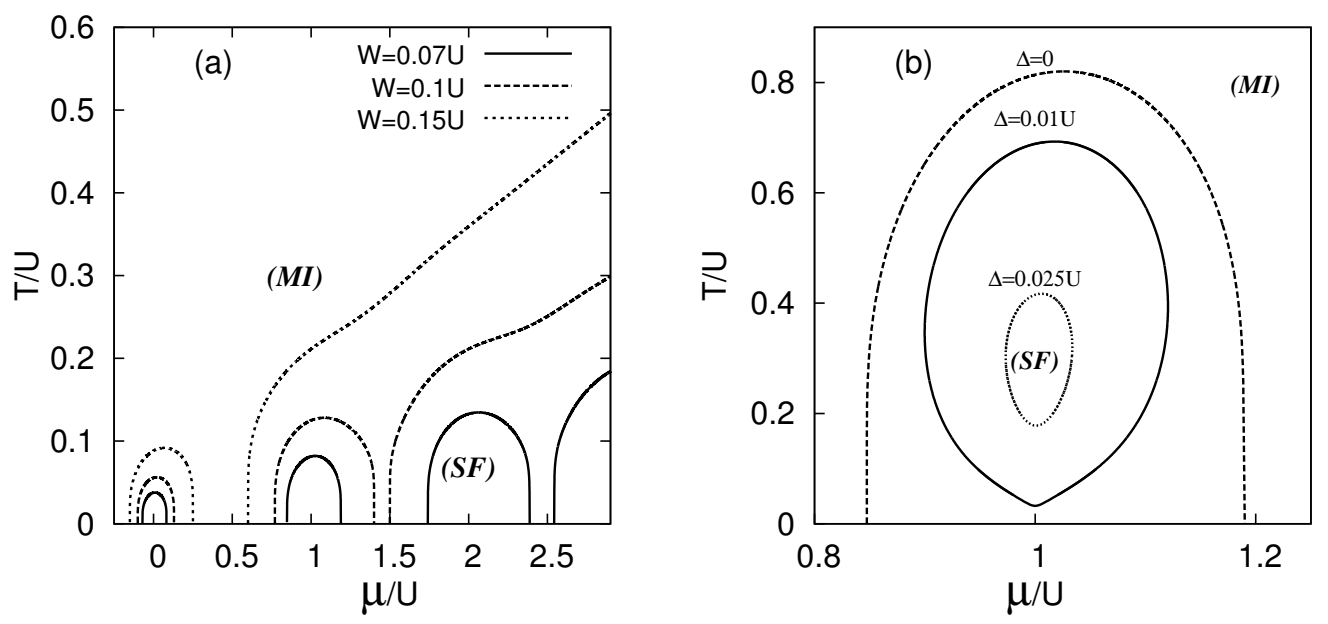

Figure 2. The $(T, \mu)$ phase diagram at $\Delta=0$ (a) and $\Delta \neq 0, W=0.07 U$ (b). 
The $(T, \mu)$ phase diagrams are given in figure 2 . The effect of thermal activation is illustrated in figure $2 \mathrm{~b}$ for the case of the one $\mathrm{SF}$ lobe $(\mu / U \approx 1)$. At the increase of the activation energy this region narrows and then the superfluid phase disappears (there is a critical value $\Delta_{\text {cr }}$ ). It should be noted that at the fixed value of the chemical potential there are two critical temperatures $T_{c_{1}}$ and $T_{c_{2}}$ at which the phase transitions between the MI and superfluid phase occur (it takes place at $\Delta \neq 0$ ). One can think that the lower critical temperature $T_{c_{2}}$ can be connected with the temperature of the transition to the superionic state in the case of superionic crystals. The simple estimate, based on the typical values of $t$ and $\Delta$ parameters for proton conductors $(\Delta \sim$ $0.1 \ldots 0.4 \mathrm{eV} ; t \sim 0.2 \ldots 0.5 \mathrm{eV})$ shows that $T_{c_{2}}$ can be of the order of several hundreds of Kelvins, which corresponds to temperatures observed in the known family $\mathrm{M}_{3} \mathrm{H}\left(\mathrm{XO}_{4}\right)_{2}\left(\mathrm{M}=\mathrm{NH}_{4}, \mathrm{Rb}, \mathrm{Cs}\right.$; $\mathrm{X}=\mathrm{S}, \mathrm{Se}$ ) of hydrogen-bonded superionic crystals [15].
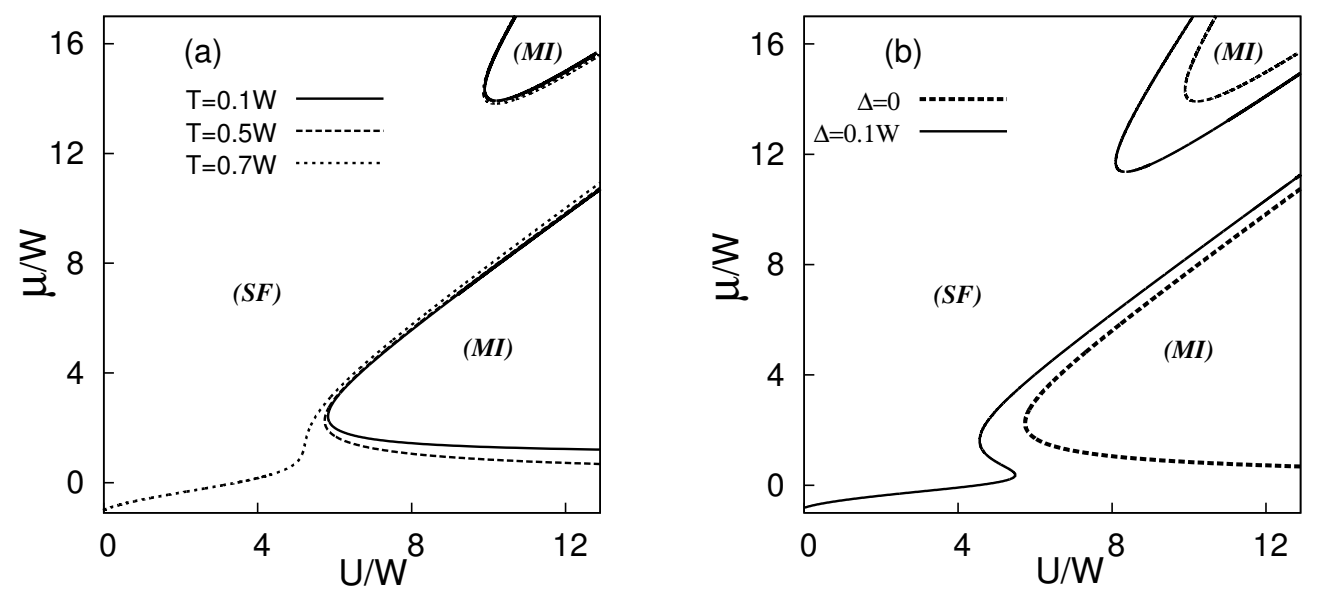

Figure 3. The $(U, \mu)$ phase diagram at $\Delta=0$ (a) and $\Delta \neq 0, T=0.5 W$ (b).

From the $(U, \mu)$ phase diagrams shown in figure 3 we can see that the effect of thermal activation on the phase diagrams is similar to the effect of temperature change in the case $\Delta=0$.

In figure 4 the $(T, \mu)$ phase diagram calculated in the independent subband approximation using the equation (20) (where $J_{0, m}=(m+1) t_{0} \exp \left(-\beta_{c}^{m} \Delta\right)$ ) is shown (dashed line). Solid line is the line shown in figure $2 \mathrm{a}$ in the case $W=0.07 U$. We can see that the independent subband approximation is applicable at small values of $m$. In this region the expression (20) adequately describes the nearly linear growth of $T_{c}^{m}$ with the increase of the number $m$.

\section{Conclusions}

The phase transitions in the Bose-Hubbard model at finite temperature are investigated. Models of such type can be applied to the description of optical lattices, ionic conductivity, intercalation in crystals, and kinetics of ionic adsorption on the crystal surfaces.

A single-particle Green's function is calculated in the random phase approximation (which is an analogy to the Hubbard-I approximation in the case of the fermionic Hubbard model) and the formalism of the Hubbard operators is used. This Green's function is obtained for the Mottinsulator and superfluid phases (in the last case we use the independent subband approximation, which is appropriate for the case of the noninteger (intermediate) mean number of the particle concentration per lattice site).

The regions of the occurrence of the superfluid and Mott insulator phases are established and the phase diagrams in the plane $(\mu, t)$ (the chemical potential - transfer parameter) are built. The effect of temperature change on this transition is analysed and the phase diagram in the $(T, \mu)$ 


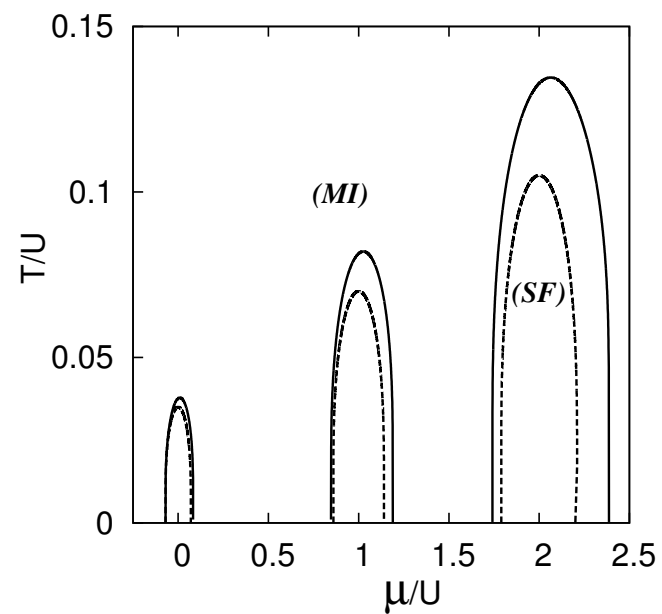

Figure 4. Dashed line denotes the phase transition line obtained in the independent subband approximation using (20). Solid line denotes the phase transition line obtained using (22). $W=$ $0.07 U, \Delta=0$.

plane is constructed. The role of thermal activation of the ion hopping is investigated by taking into account the temperature dependence of the transfer parameter $\left(t=t_{0} \exp (-\beta \Delta)\right)$. It is shown that thermal activation leads to the reconstruction of the Mott-insulator lobes (in particular, to the narrowing of the SF phase region). It is revealed that due to thermal activation of the ion hopping, there are two critical temperatures at which the MI-SF phase transition takes place (at the fixed value of the chemical potential). A simple estimation shows that the lower temperature can be quite high in proton conductors and can correspond to the temperature of the superionic phase transition in such compounds.

\section{References}

1. Greiner M., Mandel O., Esslinger T., Hänsch T.W., Bloch I., Nature, 2002, 415, 39-44.

2. Sheshadri K., Krishnamurthy H.R., Pandit R., Ramakrishnan T.V., Europhys. Lett., 1993, 22, 257263.

3. Konabe S., Nikuni T., Nakamura M., Phys. Rev. A, 2006, 73, 033621-12.

4. Ohashi Y., Kitauri M., Matsumoto H., Phys. Rev. A, 2006, 73, 033617-5.

5. Freericks J.K., Monien H., Europhys. Lett., 1994, 26, 545-550.

6. Capogrosso-Sansone B., Söyler S.G., Prokof'ev N., Svistunov B., Phys. Rev. A, 2008, 77, 015602-4.

7. Byczuk K., Vollhardt D., Phys. Rev. B, 2008, 77, 235106-14.

8. Mahan G.D., Phys. Rev. B, 1976, 14, 780-793.

9. Tomoyose T., J. Phys. Soc. Jpn., 1997, 66, 2383-2385.

10. Stasyuk I.V., Dulepa I.R., Condens. Matter Phys., 2007, 10, 259-268.

11. Mysakovych T.S., Krasnov V.O., Stasyuk I.V., Condens. Matter Phys., 2008, 11, 663-667.

12. Reilly P.D., Harris R.A., Whaley K.B., J. Chem. Phys., 1991, 95, 8599-8615.

13. Ohliger M., Pelster A. Preprint arXiv: cond-mat.stat-mech/0810.4399, 2008.

14. Stasyuk I.V., Dulepa I.R., J. Phys. Stud., 2009, 13, No. 2, 2701.

15. Stasyuk I.V., Pavlenko N., J. Phys.: Condens. Matter, 1998, 10, 7079-7090. 


\section{Фазові діаграми моделі Бозе-Хаббарда при скінченній температурі}

\section{І.В. Стасюк, Т.С. Мисакович}

Інститут фізики конденсованих систем НАН України, 79011 Львів, вул. Свєнціцького, 1

Отримано 8 липня 2009 р.

Досліджено фазові переходи в моделі Бозе-Хаббарда. Одночастинкова функція Гріна розрахована в наближенні хаотичних фаз із використанням формалізму операторів Хаббарда. Встановлено області існування надплинної фази та фази моттівського діелектрика і побудовано фазові діаграми в площині $(\mu, t)$. Проаналізовано вплив зміни температури на фазові переходи та побудовано фазові діаграми в площині $(T, \mu)$. Досліджено випадок термічної активації перескоку частинок та проаналізовано перебудову фазових діаграм у цьому випадку.

Ключові слова: модель Бозе-Хаббарда, фазові переходи

PACS: 03.75.Lm, 05.30.Jp 